\title{
Atenção Domiciliar no Brasil: estudo exploratório sobre a consolidação e uso de serviços no Sistema Único de Saúde
}

\author{
Home Care in Brazil: an exploratory study on the construction \\ process and service use in the Brazilian Health System
}

Fabiana Lima Rajão (https://orcid.org/0000-0003-3882-9992) ${ }^{1}$

Mônica Martins (https://orcid.org/0000-0002-9962-0618) ${ }^{2}$

\footnotetext{
${ }^{1}$ Programa de PósGraduação em Saúde Pública, Escola Nacional de Saúde Pública Sérgio Arouca (ENSP), Fundação Oswaldo Cruz (Fiocruz). R. Leopoldo Bulhões 1480, Manguinhos. 21041-210 Rio de Janeiro RJ Brasil. fabianarajao@gmail.com ${ }^{2}$ Departamento de Administração e Planejamento em Saúde, ENSP, Fiocruz. Rio de Janeiro RJ Brasil.
}

\begin{abstract}
Changes in demographic and epidemiological profiles, in Brazil and in the world, have brought the need to adapt the health care model. In this context, Home Care (HC) emerges as an alternative strategy of health care driven by several concerns: dehospitalization, rationalization of the hospital bed use, costs reduction, and the organization of patient-centered care. This study aims to analyze $H C$ in the Brazilian Unified Health System, identifying the modalities of care and inequalities in service use. Thereby a documentary analysis of the legislation and secondary data available on the home care volume of services and procedures explorations were realized. In total, 94,754 home-based hospitalizations occurred in the 2008-2016 period, and 4,008,692 homebased outpatient procedures were carried out in the 2012-2016 period. Outpatient HC was more widespread, while home-based hospitalizations were concentrated in some geographical areas. The regional discrepancy is striking, revealing inequalities in supply, access, and use. Despite the legal framework, the establishment of a specific program, and volume of production, HC does not seem to be yet effectively incorporated as one of the apexes of the Health Care Network.
\end{abstract}

Key words Home Care, Information Systems, Geographic Locations, Equal Access to Health Care Services
Resumo Mudanças no perfil demográfico e epidemiológico, no Brasil e no cenário internacional, fazem emergir a necessidade de adaptação do modelo de atenção em saúde. Nesse contexto, surge a Atenção Domiciliar (AD) como estratégia complementar de cuidado motivada por diversas preocupações: desospitalização, racionalização do uso de leitos hospitalares, redução de custos e organização do cuidado centrado no paciente. O objetivo deste estudo é analisar a AD no âmbito do Sistema Único de Saúde, identificando as modalidades de cuidado e desigualdades no uso. Para tal, foram realizadas análise documental da legislação e exploração de dados secundários disponiveis sobre o volume de serviços e procedimentos produzidos no domicílio. Foram contabilizadas 94.754 internações domiciliares entre 2008-2016 e 4.008.692 de procedimentos domiciliares ambulatoriais entre 2012-2016. A AD ambulatorial mostrou-se mais difundida, enquanto a internação domiciliar concentrou-se em algumas áreas geográficas. A discrepância regional é marcante, desvelando desigualdades na oferta, acesso e uso. Apesar do arcabouço legal, constituição de um programa específico e volume de produção, a $A D$ não parece, ainda, efetivamente inserida como um dos vértices da Rede de Atenção à Saúde.

Palavras-chave Assistência domiciliar, Sistemas de Informação, Localizações Geográficas, Equidade no Acesso aos Serviços de Saúde 


\section{Introdução}

Mudanças no perfil demográfico e epidemiológico, tanto no Brasil, quanto no cenário internacional, fazem emergir a necessidade de adaptação do modelo de atenção em saúde, levando diversos países a pensar na Atenção Domiciliar (AD) como um ponto de cuidado estratégico para a atenção em saúde. $\mathrm{O}$ incremento da $\mathrm{AD}$ em diversos países segue, paralelamente, ao interesse dos sistemas de saúde no processo de desospitalização, racionalização do uso de leitos hospitalares, redução de custos e organização do cuidado centrado no paciente. A demanda por serviços de $\mathrm{AD}$ surge, assim, como mais um desafio para os sistemas de saúde, contribuindo para a mudança do enfoque do cuidado e do ambiente no qual a assistência é prestada ${ }^{1}$.

O processo de envelhecimento populacional é um fator que impulsiona a preocupação dos sistemas de saúde a respeito de novos modelos de cuidado $^{1,2}$. Todavia, não é o único. A demanda por um cuidado continuado exercido em ambiente domiciliar, vem acompanhada de outras necessidades de saúde igualmente relevantes e elegíveis, como o cuidado prestado a bebês prematuros, crianças com sequelas e doenças crônicas, adultos com múltiplas doenças crônicodegenerativas, indivíduos com necessidade de cuidados paliativos, de suporte à vida e de reabilitação. Assim, a relevância da necessidade de implantação de serviços de cuidado domiciliar destaca-se na agenda sanitária atual e futura de todos os sistemas de saúde ${ }^{3}$, visando contribuir para a configuração de redes substitutivas de saúde e transformação de práticas em saúde ${ }^{2}$.

Abordando a $\mathrm{AD}$ como um modelo de cuidado, alguns autores já questionam a real necessidade de hospitalização para determinados agravos de saúde, considerando alguns motivos de internações como dispensáveis ou desnecessariamente prolongados, podendo ser substituídos ou complementados por um serviço de $\mathrm{AD}^{1,4,5}$. Em razão disso, para além da redução de gastos, a $\mathrm{AD}$ vem representando no cenário mundial a conotação de ofertar cuidado de qualidade, proporcionando bem-estar e conforto ao permitir a permanência do doente em seu ambiente domiciliar, integrado ao seu contexto de vida ${ }^{5}$.

O trabalho em saúde no domicílio não trata apenas de delegar à família a função da assistência prestada ao seu familiar. Para Kerber et al. ${ }^{4}$, ao reconhecer o ambiente domiciliar como próprio ao cuidado, como um espaço de ação, de execução do trabalho em saúde, o setor saúde veicula a presença do Estado e colabora com a expansão de seus serviços, por meio de um alcance maior de suas ações. Contudo, isso aporta particularidades, pois a prática do cuidado domiciliar pressupõe a interação de três atores: o paciente (usuário), o cuidador e o profissional de saúde. A integração entre sistema de saúde e usuário na execução do cuidado em saúde em domicílio é configurada pelo serviço de saúde, serviço este executado por uma equipe multidisciplinar, que presta o cuidado necessário ao usuário com o acompanhamento de um cuidador, por sua vez representado por um familiar, amigo ou vizinho ${ }^{6}$. $\mathrm{O}$ cuidador transita entre a função de prestador do cuidado e também de usuário, já que, simultaneamente, desempenha práticas do cuidado, demanda apoio técnico, emocional e social dos profissionais de saúde. Uma questão a ser levada em consideração, diz respeito ao cuidador familiar eleito, na maioria das vezes, ser representado por mulheres, papel comum a esposa ou filha. Nessa ótica, a tarefa de cuidar associada à figura feminina tem raízes históricas, culturais, sociais e afetivas; ou seja, é tradicionalmente responsabilidade da mulher o trabalho de casa, crianças, idosos ou doentes, vista como uma cuidadora natural ${ }^{7,8}$.

Ademais, no cuidado domiciliar o profissional precisará inserir-se no contexto cultural e familiar do doente, por esse motivo necessitará de sensibilidade para compreender amplamente como o cuidado será realizado e quais entraves. Nesse sentido, o trabalho em saúde não se restringe apenas ao cuidado biológico, mas abarca a dimensão das interações entre a vida humana e o ambiente ${ }^{4}$. Para Kerber et al. ${ }^{4}$, a AD pode ser considerada um instrumento potencial para a promoção de uma assistência moldada pela relação e vínculo entre trabalhador e usuário, pelo acolhimento e desenvolvimento de co-responsabilidades. Há ainda, necessidade de integração das tecnologias em saúde, com relevância de tecnologias leves, considerando que a relação profissional-doente-família ocorre no lar do enfermo, diferente de uma internação hospitalar, em que a família e o doente precisam se adaptar, se ajustar ao meio, à instituição, à prática de trabalho estabelecida, diante de regras e normas específicas ${ }^{2}$. Assim, quando a prática do cuidado em saúde é deslocada para o ambiente domiciliar, o esforço de ajuste a uma dada realidade cultural e familiar deve ser desempenhado pelo profissional de saúde, de forma que o aspecto relacional se torna relevante para o sucesso da assistência, onde pacientes e cuidadores, em suas casas, desem- 
penham um papel muito mais ativo e assumem muitas responsabilidades que são, em outros ambientes, de responsabilidade restrita aos profissionais de saúde $\mathrm{e}^{9-11}$.

Conceitualmente, a AD é classificada em quatro modalidades de cuidado, que se diferenciam pela especificidade de suas finalidades e ações, são elas: a atenção domiciliar, atendimento domiciliar, visita domiciliar e internação domiciliar ${ }^{12}$ (Quadro 1). Há variabilidade na conceituação, mas também delimitadores consensuais (Quadro 1). Nesta perspectiva Lacerda et al. ${ }^{12}$ destacou que os conceitos e modalidades de cuidado inerentes à assistência em saúde prestada no domicílio variam entre autores, em um certo sentido retratam distinções não somente conceituais, mas também do ponto de vista prático em termos da organização desse tipo de cuidado, havendo uma complementariedade e interdependência entre as quatro modalidades conceituadas. Em termos gerais, a nomenclatura 'Atenção Domiciliar' designa um conceito de maior amplitude, um termo geral que designa a organização do sistema e das práticas de saúde, que abrange o atendimento, a visita e a internação domiciliar. O atendimento domiciliar é também considerado como assistência domiciliar ou cuidado domiciliar, na literatura internacional: home care. A visita domiciliar é, por sua vez, o conceito mais difundido no sistema de saúde brasileiro e nas práticas de saúde da comunidade. $\mathrm{E}$ a internação domiciliar, modalidade da atenção domiciliar que se utiliza das tecnologias duras em saúde, relacionada coma prestação de serviços de média e alta complexidade.

A AD, já presente no cenário internacional, desponta como uma demanda iminente também para o sistema de saúde nacional. Desta forma, visando elucidar como este modelo de cuidado se insere no contexto nacional, o presente trabalho objetivou analisar a AD no âmbito do SUS, de forma a identificar a legislação, o processo de consolidação, as modalidades de cuidado, os recursos disponíveis, além de caracterizar sua utilização e dimensionar desigualdades no uso de serviços no contexto nacional.

\section{Metodologia}

\section{Desenho do estudo}

Estudo exploratório com abordagem qualitativa e quantitativa, apoiado na literatura científica, documentos oficiais e dados secundários para a descrição da $\mathrm{AD}$ no território nacional, com- preensão do seu papel e processo de consolidação no SUS.

\section{Fonte de informação}

Revisão bibliográfica foi realizada para obter experiências, inciativas e avaliações sobre o tema, para tal foram empregados os seguintes descritores: atenção domiciliar, assistência domiciliar, internação domiciliar, atendimento domiciliar e cuidado domiciliar. Buscou-se artigos científicos em português, inglês e espanhol, publicados no período de 1995 a 2018, nas bases bibliográficas: BVS, Scielo e PubMed. Foram realizadas também consultas com base nas citações bibliográficas de cada artigo, teses, dissertações, e relatórios de pesquisa.

Pesquisas documentais, incluindo leis, resoluções, portarias e manuais também compuseram essa revisão. Para início desta etapa foram lidas as primeiras portarias encontradas nas referências bibliográficas, que foram levando ao conhecimento das demais. Consultas na plataforma do Ministério da Saúde, Saúde Legis, com buscas a partir da nomenclatura domiciliar, permitiram identificar um enredo de portarias subsequentes. Manuais instrutivos e demais documentos oficiais foram utilizados para conhecimento da organização da AD no SUS no período estudado.

Inicialmente, para identificação de serviços ofertados e procedimentos de saúde produzidos pelo SUS no âmbito da AD, foram consultados a Relação Nacional de Ações e Serviços de Saúde do SUS (RENASES) e o Sistema de Gerenciamento da Tabela de Procedimentos, Órteses, Próteses e Medicamentos do SUS (SIGTAP/SUS). A consulta à RENASES permitiu a identificação de 5 tipos de ações e serviços no âmbito da AD: Atenção Domiciliar; Atenção Familiar; Visita Domiciliar ou Institucional; Assistência Domiciliar Terapêutica Multiprofissional em HIV/AIDS (ADTM); e Assistência Especializada Domiciliar Realizada por Equipe Multiprofissional. A consulta ao SIGTAP/SUS permitiu identificar 17 procedimentos realizados em ambiente domiciliar pelo SUS: 01.01.03.001-0, 01.01.03.002-9, 03.01.01.013-7, 03.01.01.016-1, 03.01.05.001-5, 03.01.05.002-3, 03.01.05.003-1, 03.01.05.004-0, 03.01.05.006-8, 03.01.05.006-6, 03.01.05.007-4, 03.01.05.010-4, 03.01.05.014-7, 03.01.08.024-0, 03.05.01.016-6, 07.02.10.004-8, 07.02.10.005-6, suas formas de execução, níveis de complexidade, tipos de financiamento e disponibilidade nos Sistemas de Informação em Saúde do SUS (SIS-SUS). Ambos orientaram a busca nos sistemas de informação para construção da base de dados utilizada. 
Quadro 1. Conceituação sobre a assistência em saúde prestada no ambiente domiciliar.

\begin{tabular}{|c|c|}
\hline \multicolumn{2}{|r|}{ Conceitos } \\
\hline \multicolumn{2}{|r|}{ Atenção Domiciliar } \\
\hline Brasil $(2006)^{22}$ & $\begin{array}{l}\text { Termo genérico que envolve ações de promoção à saúde, prevenção, tratamento de doenças e } \\
\text { reabilitação, realizados em domicílio. }\end{array}$ \\
\hline $\begin{array}{l}\text { Lacerda } \\
(2006)^{12}\end{array}$ & $\begin{array}{l}\text { A atenção domiciliar envolve a prática de políticas econômicas, sociais e de saúde, para } \\
\text { reduzir os riscos de os indivíduos adoecerem; a fiscalização e o planejamento dos programas } \\
\text { de saúde; e a execução das atividades assistenciais, preventivas e educativas. Assim, abrange } \\
\text { desde a promoção até a recuperação dos indivíduos acometidos por um agravo e que estão } \\
\text { sediados em seus respectivos lares. }\end{array}$ \\
\hline $\begin{array}{l}\text { Word Health } \\
\text { Organization } \\
(\text { EUA, 1999) }\end{array}$ & $\begin{array}{l}\text { Para o sistema americano, a atenção domiciliar à saúde pode ser compreendida como "home } \\
\text { healthcare", sendo o cuidado à saúde proporcionado às pessoas no próprio domicílio de } \\
\text { forma apropriada e de alta qualidade, com relação ao custo-benefício compatível com a vida } \\
\text { dos indivíduos, que devem manter sua autonomia, independência e melhor qualidade de vida. }\end{array}$ \\
\hline \multicolumn{2}{|r|}{ Atendimento Domiciliar } \\
\hline $\operatorname{Brasil}(2016)^{24}$ & $\begin{array}{l}\text { Assistência domiciliar (ou atendimento domiciliar) é um conjunto de atividades de caráter } \\
\text { ambulatorial, programadas e continuadas, desenvolvidas em domicílio. }\end{array}$ \\
\hline $\begin{array}{l}\text { Wealth Canada } \\
(1997)^{25}\end{array}$ & $\begin{array}{l}\text { Provisão de uma disposição de serviços de saúde e sociais designados a dar suporte aos } \\
\text { pacientes em suas próprias casas. }\end{array}$ \\
\hline $\begin{array}{l}\text { Ryu et al. } \\
(\text { Coreia, } \\
2004)^{26}\end{array}$ & $\begin{array}{l}\text { "Home care" baseado em uma perspectiva do setor público de saúde, por meio de ações de } \\
\text { saúde realizadas no domicílio, junto à família e à comunidade, destinadas aos pacientes com } \\
\text { doenças crônicas e com necessidade de longos períodos de tratamento, evitando a ocorrência } \\
\text { de hospitalização. }\end{array}$ \\
\hline $\begin{array}{l}\text { Thomé et } \\
\text { al. (Suécia, } \\
2003)^{27}\end{array}$ & $\begin{array}{l}\text { Cuidado prestado por profissionais de saúde ao enfermo em sua própria casa, com objetivo } \\
\text { final de bem-estar, contribuindo para a qualidade de vida e para a melhoria do estado de } \\
\text { saúde, de forma a substituir o cuidado hospitalar. }\end{array}$ \\
\hline \multicolumn{2}{|r|}{ Visita Domiciliar } \\
\hline $\begin{array}{l}\text { Ribeiro } \\
(2004)^{28}\end{array}$ & $\begin{array}{l}\text { A visita domiciliar caracteriza-se pelo contato pontual de profissionais de saúde com as } \\
\text { populações de risco, enfermos e seus familiares para a coleta de informações e/ou orientações. }\end{array}$ \\
\hline Mazza $(2004)^{29}$ & $\begin{array}{l}\text { Na visita domiciliar são desenvolvidas ações de orientação, educação, levantamento de } \\
\text { possíveis soluções de saúde, fornecimento de subsídios educativos, para que os indivíduos } \\
\text { atendidos tenham condições de se tornar independentes. }\end{array}$ \\
\hline $\begin{array}{l}\text { Freitas et al. } \\
(2000)^{30}\end{array}$ & $\begin{array}{l}\text { A visita domiciliar deve compreender ações sistematizadas, que se iniciam antes da visita e } \\
\text { continuam após ela. }\end{array}$ \\
\hline $\begin{array}{l}\text { Murashima } \\
\text { et al. (Japão, } \\
2002)^{31}\end{array}$ & $\begin{array}{l}\text { Atividades de visita domiciliar realizadas por enfermeiras, que após o primeiro contato com } \\
\text { a família definem as abordagens do cuidado, assim como as orientações a serem repassadas } \\
\text { ao enfermo e à família para a realização das atividades de vida diária. Permite a provisão de } \\
\text { um cuidado individualizado ao paciente, que pode ser um idoso, pacientes que necessitam de } \\
\text { cuidados especiais e de tecnologia avançada, pacientes terminais ou pacientes com doenças } \\
\text { mentais. }\end{array}$ \\
\hline \multicolumn{2}{|r|}{ Internação Domiciliar } \\
\hline $\begin{array}{l}\text { Ribeiro } \\
(2004)^{28}\end{array}$ & $\begin{array}{l}\text { A internação domiciliar é uma atividade continuada, com oferta de tecnologia e de recursos } \\
\text { humanos, equipamentos, materiais e medicamentos, para pacientes em estados mais } \\
\text { complexos, que demandam assistência semelhante à oferecida em ambiente hospitalar. }\end{array}$ \\
\hline $\begin{array}{l}\text { Lacerda } \\
(2000)^{32}\end{array}$ & $\begin{array}{l}\text { A internação domiciliar é a prestação de cuidados sistematizados de forma integral e contínua } \\
\text { no domicílio, com supervisão e ação da equipe de saúde específica, personalizada, centrada na } \\
\text { realidade do cliente, envolvendo a família e, podendo ou não, utilizar equipamentos e materiais. }\end{array}$ \\
\hline $\begin{array}{l}\text { Nogueira } \\
(\text { França, } \\
2000)^{33}\end{array}$ & $\begin{array}{l}\text { A internação é uma alternativa assistencial do setor saúde que consiste em um modelo } \\
\text { organizado capaz de dispensar um conjunto de cuidados e atenção de médicos, enfermeiras, } \\
\text { tanto em qualidade quanto em quantidade, para pacientes em seu domicílio, que não precisam } \\
\text { de uma infraestrutura hospitalar, mas precisam de vigilância ativa e assistência completa. }\end{array}$ \\
\hline
\end{tabular}

Dados secundários foram obtidos nos sistemas de informação de uso público e irrestrito, anônimos, sendo acessados por meio da pla- taforma do Departamento de Informática do SUS (DATASUS). No primeiro momento, buscou-seinformações preliminares por meio de 
tabulação on-line (Tabnet), utilizando dados do Sistema de Informação em Saúde para a Atenção Básica (SISAB) no sentido de verificar a existência de registros referentes aos cuidados prestados em domicílio. Nesta etapa, foi excluída a possibilidade de uso do SISAB, visto que tal sistema de informação não permitia, naquele momento, acesso público aos microdados.

Com base na busca orientada pelos portais da RENASES e SIGTAP, utilizou-se como fonte de dados os seguintes SIS-SUS: Sistema de Informações Hospitalares do SUS (SIH-SUS) e Sistema de Informações Ambulatoriais do SUS (SIA-SUS). Foram extraídos do SIH-SUS todos os registros dos arquivos do tipo reduzido, cuja variável procedimento realizado correspondia ao código da internação domiciliar: 03.01.05.007-4. Esses registros conformaram a base de dados sobre as internações domiciliares realizadas em âmbito nacional no período de estudo, esta base foi extraída com todas as variáveis disponíveis para cada internação. Do SIA-SUS foram extraídos os dados da produção ambulatorial da Atenção Domiciliar, cuja variável procedimento correspondia aos 17 códigos de atendimento ambulatorial domiciliar. Em ambos sistemas de informação os arquivos de cada uma das Unidades da Federação foram importados, filtrados e, posteriormente, tabulados por meio do programa de tabulação e tratamento de dados do DATASUS, TabWin. Após importadas do DATASUS, as bases de dados nacionais foram contruídas separadamente no TabWin.

\section{Universo de estudo}

O universo do estudo incluiu as internações domiciliares e os procedimentos ambulatoriais de âmbito domiciliar, respectivamente encontrados nos sistemas de informação SIH-SUS e SIA-SUS. Buscou-se explorar o período de tempo disponível nos SIS-SUS, sendo este possível a partir de 2008 no SIH-SUS e a partir de novembro de 2012 no SIA-SUS. Vale destacar que a cobertura temporal de cada sistema tem relação com o próprio processo de conformação da $\mathrm{AD}$, em concordância com a deliberação das portarias correspondentes aos registros das produções nas respectivas bases.

O recorte temporal considerou o período disponível de cada SIS-SUS, escolhendo utilizar os dados processados até 2016, devido a data de início deste estudo, rotina de revisão e correção das informações disponibilizadas em curso para o ano de 2017. Dessa forma, foram analisadas as seguintes informações: SIH-SUS (janeiro de 2008 a dezembro de 2016) e SIA-SUS (novembro de 2012 a dezembro de 2016). Assim, o recorte temporal não foi o mesmo para ambos os bancos, já que a pesquisa objetivou explorar as informações disponíveis nas bases de dados do SUS, não havendo a pretensão de comparação de variáveis entre as bases.

Ao fim do processo de extração de dados, o universo de estudo contabilizou 94.754 internações domiciliares no período de 2008 a 2016, bem como um total de 4.008 .692 de procedimentos domiciliares ambulatoriais no período de novembro de 2012 a 2016.

\section{Análise dos dados}

Pelo caráter exploratório do estudo, a análise dos dados foi circunscrita a etapa descritiva. $\mathrm{Na}$ construção dos bancos de dados, processamento dos dados, elaboração das tabelas, gráficos e mapas foram utilizados os programas: TabWin (DATASUS), SPSS 17.0 (SPSS Inc.,Chicago, EUA) e Microsoft Excel 2010.

As variáveis analisadas foram aquelas disponíveis nos SIS-SUS, quais sejam: o ano de atendimento/internação, sexo, idade, cor/raça, diagnóstico, local do atendimento/internação (localização geográfica de residência), origem do paciente (atenção básica, emergência, internação hospitalar), destino ou motivo de saída do atendimento/internação (alta, permanência, reinternação e óbito), dias de permanência sob atendimento/internação domiciliar, e tipo de gestão (público, privado e filantrópico).Tais variáveis foram inicialmente exploradas no Excel e, posteriormente, a análise descritiva foi realizada no SPSS.

Por fim, ensejou-se relacionar as informações quantitativas com marcos identificados na análise documental e, com isso, compreender a conformação atual da AD no SUS.

\section{Considerações éticas}

Além da literatura científica, foram empregados como fonte de informação documentos e base de dados secundários de domínio público e irrestrito. Portanto, isento de parecer, de acordo com as resoluções da Comissão Nacional de Ética em Pesquisa (CONEP). 


\section{Resultados e discussão}

\section{Legislação e processo de consolidação da AD}

O processo de consolidação da AD no SUS está ancorado em diversas normatizações dessa modalidade ao longo dos últimos 30 anos. No total, somam-se 19 portarias que tratam da organização e efetivação de serviços relacionados com a AD: a primeira deliberada em 1998 e a mais recente em $2016^{13}$. Paralelamente às portarias que formaram o arcabouço da AD no SUS, foram deliberadas outras 70 portarias que trataram especificamente de habilitar e desabilitar Serviços de Atenção Domiciliar (SAD) em diversos municípios. Ao longo da criação das referidas portarias, foram também organizados quatro manuais instrutivos que, de maneira mais prática, explicitaram a organização atual do processo de trabalho da AD no SUS ${ }^{6,14-16}$.

Como a primeira legislação voltada para a organização dos serviços de saúde prestados no domicílio, tem-se a publicação, em 1998, da Portaria $\mathrm{n}^{\circ} 2.416$, que estabeleceu requisitos para credenciamento de hospitais e critérios para realização de internação domiciliar no SUS. A partir daí a configuração da AD no SUS foi ocorrendo gradativamente por meio da formulação de portarias subsequentes e complementares. Em 2001, foi instituída a Portaria no 1.531 no intuito de oferecer aos portadores de distrofia muscular progressiva o uso de ventilação mecânica não invasiva (VNI) em domicílio, sob cuidado de uma equipe multidisciplinar, financiada pelo SUS. Vale salientar que, em 2008, a Portaria no 370 ampliou o escopo de atuação deste programa, incluindo novas patologias elegíveis para o seu cadastramento. Em 2002, a Portaria no 249, estabeleceu a assistência domiciliar como uma modalidade a ser desenvolvida pelo Centro de Referência em Assistência à Saúde do Idoso. A Resolução da Diretoria Colegiada da Agência Nacional de Vigilância Sanitária (RDC - ANVISA) no 11 , foi deliberada em 2006, e dispôs sobre o regulamento técnico do funcionamento dos serviços que prestam assistência domiciliar ${ }^{13}$. A estruturação dos então denominados $\mathrm{SAD}$, passa a basear-se na orientação dessa resolução.

Em 2006, o Ministério da Saúde (MS) publicou a Portaria $n^{\circ} 2.529$, que instituiu a internação domiciliar no âmbito do SUS e a definiu como um conjunto de atividades prestadas às pessoas clinicamente estáveis, em seu domicílio, cuja condição de saúde exigisse uma intensidade de cuidado superior às modalidades ambulatoriais, mas que pudessem ser mantidas em casa por meio da presença de uma equipe de profissionais exclusivos para este fim. Cinco anos depois, em agosto de 2011, tal portaria foi revogada e uma nova Portaria $n^{\circ} 2.029$, talvez a mais importante, representou um marco legislativo no processo de estruturação da $\mathrm{AD}$, por abordá-la como uma modalidade de atenção no âmbito do SUS, de forma a retirar o foco da internação domiciliar como modelo único de prestação da assistência domiciliar, fazendo menção à $\mathrm{AD}$ como uma incorporação tecnológica de caráter substitutivo ou complementar à intervenção hospitalar.

Data de 8 de novembro de 2011 o lançamento do Programa Melhor em Casa, pelo governo federal, que incorporou a $\mathrm{AD}$ ao SUS como um dos componentes das Redes de Atenção às Urgências e Emergências (RUE) e, nessa perspectiva, de acordo com a proposição da Portaria $\mathrm{n}^{\circ} 1.600$, de julho de 2011, deveria estar inserida nas Redes de Atenção à Saúde (RAS). Dessa forma, segundo as diretrizes do Programa, a AD constitui-se no SUS como uma modalidade de atenção à saúde substitutiva ou complementar, caracterizada por um conjunto de ações de promoção à saúde, prevenção, tratamento de doenças e reabilitação, prestadas em domicílio, em busca de um cuidado ampliado e continuado, integrado com as RAS.

Quanto a responsabilização pela execução da $\mathrm{AD}$, a Portaria $\mathrm{n}^{\circ}$ 2.527, em 2011, reestabeleceu normas para cadastro dos SAD e suas respectivas equipes, assim denominadas: Equipes Multidisciplinares de Atenção Domiciliar (EMAD) e Equipes Multidisciplinares de Apoio (EMAP). Também estabeleceu critérios de habilitação dos estabelecimentos de saúde, como um SAD, aos quais as EMAD e EMAP estariam vinculadas. Em seguida, em 2013, a Portaria no 963 novamente redefiniu a $\mathrm{AD}$ no âmbito do SUS; ganhando nova versão em 2016, a partir da Portaria no 825 que, além de redefinir a $\mathrm{AD}$ no âmbito do SUS, impulsionou a habilitação dos SAD.

A partir de 2011, paralelamente às portarias que estruturaram a $\mathrm{AD}$ no SUS, foram sendo deliberadas portarias que habilitavam SAD, contemplando municípios com suas respectivas equipes multiprofissionais, as EMAD e EMAP. No decorrer de sete anos, desde a deliberação da primeira portaria de habilitação, até o presente ano, em 2018, 70 portarias foram deliberadas, contemplando 651 municípios, com 1.000 estabelecimentos de saúde habilitados como SAD (dentre esses UPAs, hospitais e UBSs), estando compostos por 1.361 EMAD e 727 EMAP. 


\section{Volume de produção e utilização de serviços e procedimentos relativos à $\mathrm{AD}$}

Na busca realizada nas bases dos SIS-SUS, no SIH-SUS por internações domiciliares e no SIA-SUS por procedimentos domiciliares ambulatoriais, foram encontrados 97.754 registros de internações domiciliares no período de 2008 a 2016 e 4.008 .612 registros referentes a procedimentos ambulatoriais realizados no domicílio, no período de 2012 a 2016 (Tabela 1). Em virtude de terem bases de dados e períodos de registro distintos, não foi intenção desse trabalho comparar as modalidades da atenção domiciliar.

Em relação à distribuição das internações domiciliares, no período de estudo foi percebido um aumento desse registro a partir de 2010
(Tabela 1). Apesar da Portaria que estabeleceu a internação domiciliar no âmbito do SUS ter sido deliberada em 2006, os registros dessa atividade só ficaram disponíveis a partir de 2008 no SIHSUS. O ano de 2011 apresentou o maior percentual de registros $(16,3 \%)$, que pode ter relação com o período de conformação da $\mathrm{AD}$ como modelo de Atenção do SUS, neste mesmo ano.

Quanto aos procedimentos domiciliares ambulatoriais, os primeiros registros constam no ano de 2012, a partir do mês de novembro, o que parece justificar o menor percentual $(4,2 \%)$ neste ano (Tabela 1). Um ano antes, foi deliberada a Portaria no 2.029 que resolveu a AD como modelo de atenção e criou o Programa Melhor em Casa. Nos anos subsequentes houve uma crescente no número desses registros, sendo maior em

Tabela 1. Perfil de utilização da atenção domiciliar. Brasil, 2008-2016.

\begin{tabular}{|c|c|c|c|c|c|}
\hline & \multirow[t]{2}{*}{ Características* } & \multicolumn{2}{|c|}{$\begin{array}{l}\text { Internações } \\
\text { Domiciliares }\end{array}$} & \multicolumn{2}{|c|}{$\begin{array}{c}\text { Assistência Domiciliar } \\
\text { Ambulatorial }\end{array}$} \\
\hline & & $\mathrm{n}$ & $\%$ & $\mathrm{n}$ & $\%$ \\
\hline & Total & 95.754 & 100,00 & 4.008 .612 & 100,00 \\
\hline \multicolumn{6}{|l|}{ Ano } \\
\hline 2008 & & 4.824 & 5,0 & - & - \\
\hline 2009 & & 6.377 & 6,7 & - & - \\
\hline 2010 & & 11.242 & 11,7 & - & - \\
\hline 2011 & & 15.606 & 16,3 & - & - \\
\hline 2012 & & 12.577 & 13,1 & 167.965 & 4,2 \\
\hline 2013 & & 10.181 & 10,6 & 622.596 & 15,5 \\
\hline 2014 & & 10.315 & 10,8 & 1.318 .271 & 32,9 \\
\hline 2015 & & 12.177 & 12,7 & 1.706 .196 & 42,6 \\
\hline 2016 & & 12.455 & 13,0 & 193.584 & 4,8 \\
\hline \multicolumn{6}{|l|}{ Sexo } \\
\hline Masculino & & 46.779 & 48,9 & 1.874 .056 & 46,8 \\
\hline Feminino & & 48.975 & 51,1 & 2.128 .264 & 53,1 \\
\hline Ignorado & & - & - & 6.292 & 0,2 \\
\hline \multicolumn{6}{|l|}{ Faixa etária } \\
\hline 0-29 dias & & 194 & 0,2 & 41.136 & 1,0 \\
\hline 1-4 anos & & 3.915 & 4,1 & 43.185 & 1,1 \\
\hline 5-9 anos & & 2.140 & 2,2 & 37.475 & 0,9 \\
\hline 10-19 anos & & 4.121 & 4,3 & 119.176 & 3,0 \\
\hline 20-29 anos & & 4.558 & 4,8 & 183.586 & 4,6 \\
\hline 30-39 anos & & 5.147 & 5,4 & 211.446 & 5,3 \\
\hline $40-49$ anos & & 7.477 & 7,8 & 257.696 & 6,4 \\
\hline 50-59 anos & & 11.590 & 12,1 & 424.504 & 10,6 \\
\hline $60-69$ anos & & 16.001 & 16,7 & 641.798 & 16,0 \\
\hline 70-79 anos & & 19.615 & 20,5 & 896.533 & 22,4 \\
\hline $80-89$ anos & & 15.947 & 16,7 & 837.552 & 20,9 \\
\hline 90-99 anos & & 4.693 & 4,9 & 250.606 & 6,3 \\
\hline 100 e mais & & 356 & 0,4 & 19.496 & 0,5 \\
\hline
\end{tabular}


Tabela 1. Perfil de utilização da atenção domiciliar. Brasil, 2008-2016.

\begin{tabular}{|c|c|c|c|c|}
\hline \multirow[t]{2}{*}{ Características ${ }^{\star}$} & \multicolumn{2}{|c|}{$\begin{array}{l}\text { Internações } \\
\text { Domiciliares }\end{array}$} & \multicolumn{2}{|c|}{$\begin{array}{c}\text { Assistência Domiciliar } \\
\text { Ambulatorial }\end{array}$} \\
\hline & $\mathbf{n}$ & $\%$ & $\mathbf{n}$ & $\%$ \\
\hline Total & 95.754 & 100,00 & 4.008.612 & 100,00 \\
\hline \multicolumn{5}{|l|}{ Cor/Raça } \\
\hline Branca & 19.532 & 20,4 & 1.588 .032 & 39,6 \\
\hline Preta & 2.426 & 2,5 & 234.324 & 5,8 \\
\hline Parda & 18.009 & 18,8 & 1.072 .427 & 26,8 \\
\hline Amarela & 1.264 & 1,3 & 33.798 & 0,8 \\
\hline Indígena & 19 & 0,0 & 318 & 0,0 \\
\hline Sem informação & 54.504 & 56,9 & 1.079 .713 & 26,9 \\
\hline \multicolumn{5}{|l|}{ Diagnóstico principal (Capítulo CID - 10) } \\
\hline II. Neoplasmas (tumores) & 8.939 & 9,3 & 218.992 & 5,5 \\
\hline IV. Doenças endócrinas,nutricionais e metabólicas & 7.695 & 8,0 & 182.402 & 4,6 \\
\hline V. Transtornos mentais e comportamentais & - & - & 123.736 & 3,1 \\
\hline VI. Doenças do sistema nervoso & 11.679 & 12,2 & 601.248 & 15,0 \\
\hline IX. Doenças do aparelho circulatório & 27.392 & 28,6 & 1.239 .149 & 30,9 \\
\hline X. Doenças do aparelho respiratório & 20.874 & 21,8 & 179.329 & 4,5 \\
\hline XII. Doenças da pele e do tecido subcutâneo & 6.243 & 6,5 & 206.346 & 5,1 \\
\hline $\begin{array}{l}\text { XIII. Doenças do sistema osteomuscular e do tecido } \\
\text { conjuntivo }\end{array}$ & - & - & 95.151 & 2,4 \\
\hline XIX. Lesões por envenenamento e outras causas externas & 11.064 & 11,6 & 390.294 & 9,7 \\
\hline Outros & 1.868 & 2,0 & 358.234 & 8,9 \\
\hline CID inválido ou não identificado & - & - & 44 & 0,0 \\
\hline Diferença/inconsistência & - & - & 413.687 & 10,3 \\
\hline \multicolumn{5}{|l|}{ Resultado do cuidado } \\
\hline Alta & 30.423 & 31,8 & 211.974 & 5,3 \\
\hline Permanência & 50.872 & 53,1 & 3.577 .290 & 89,2 \\
\hline Transferência & 2.548 & 2,7 & - & - \\
\hline Encaminhamento à $\mathrm{AD} 1$ & - & - & 93.820 & 2,3 \\
\hline Admissão na Urgência & - & - & 13.028 & 0,3 \\
\hline Internação hospitalar & & & 37.741 & 0,9 \\
\hline Encerramento administrativo & 10.340 & 10,8 & - & - \\
\hline Óbito & 1.571 & 1,6 & 68.280 & 1,7 \\
\hline Não informado & - & - & 6.479 & 0,2 \\
\hline \multicolumn{5}{|l|}{ Região geográfica de residência } \\
\hline Norte & 1.208 & 1,3 & 155.930 & 3,9 \\
\hline Nordeste & 43.324 & 45,2 & 1.042 .978 & 26,0 \\
\hline Sudeste & 21.591 & 22,5 & 2.136 .830 & 53,3 \\
\hline Sul & 12.064 & 12,6 & 457,637 & 11,4 \\
\hline Centro-Oeste & 17.567 & 18,3 & 208.945 & 5,2 \\
\hline Ignorado ou exterior & - & - & 6.292 & 0,2 \\
\hline
\end{tabular}

${ }^{\star}$ Apresentadas somente as variáveis comuns às bases de dados consultadas.

Fonte: Ministério da Saúde: Sistema de Informações Hospitalares (SIH/SUS) - Movimento de AIH, Sistema de Informações Ambulatoriais (SIA/SUS) - Serviço de Atenção Domiciliar.

2015 (42,6\%), porém reduzido em 2016 (4,8\%) (Tabela 1), que pode ter relação com o processo de desabilitação dos SAD, verificado em Portarias específicas, por motivos diversos. Isto, certamen- te, merece um estudo minucioso em relação à desabilitação de SAD, que possam vir a explicar essa situação ou mesmo confiabilidade da informação. 
Em ambas as modalidades de $\mathrm{AD}$, não houve diferença significativa entre os sexos (Tabela 1). Sendo ligeiramente maior a utilização dos serviços por mulheres, correspondendo a $51,1 \%$ nas internações domiciliares e $53,1 \%$ nos procedimentos domiciliares ambulatoriais.

Igualmente, o aumento do volume de utilização de AD foi proporcional ao avançar das idades (Tabela 1). A utilização por crianças e indivíduos mais jovens não mostrou-se significativa. A partir dos 50 anos, nas duas modalidades, é percebido o aumento da utilização pelos serviços. A utilização por indivíduos a partir de 60 anos correspondeu a mais da metade de todos os serviços e procedimentos utilizados (Tabela 1). Informação essa que vai ao encontro do destaque dado pela literatura a esta faixa, quando abordado os objetivos e a quem se destina a $\mathrm{AD}^{17,18}$. A faixa etária, comparativamente, que mais utilizou a $\mathrm{AD}$ foi a de 70 a 79 anos, correspondendo a $20,5 \%$ das internações domiciliares, e 22,4\% dos procedimentos domiciliares ambulatoriais (Tabela 1).

A utilização por indivíduos de cor/raça branca foi predominante, correspondendo a $20,4 \%$ nas internações domiciliares e a 39,6\% nos atendimentos domiciliares ambulatoriais; seguido dos indivíduos de cor/raça parda, $18,8 \%$ nas internações domiciliares e $26,8 \%$ dentre os procedimentos domiciliares ambulatoriais. Chamou atenção a ausência desta informação em ambas modalidades, principalmente nas internações domiciliares, correspondendo a $56,9 \%$ dos registros, e $26,9 \%$ nos procedimentos domiciliares ambulatoriais (Tabela 1).

A maior parte dos pacientes que utilizaram o serviço de atenção domiciliar, tiveram como diagnóstico principal, segundo Capítulo da Classificação Internacional de Doenças e Problemas Relacionados com a Saúde-10a revisão (CID10), as doenças do aparelho circulatório, $28,6 \%$ nas internações domiciliares e $30,9 \%$ nos procedimentos domiciliares ambulatoriais (Tabela 1). As doenças do aparelho respiratório foram a causa de $21,8 \%$ das internações domiciliares, mas corresponderam a apenas $4,5 \%$ dos procedimentos domiciliares ambulatoriais; talvez pela complexidade desse cuidado, justificando o uso de tecnologias mais duras, assim caracterizando uma internação com uso de tecnologias de média e alta complexidade, em detrimento de práticas ambulatoriais. As doenças do sistema nervoso foram equivalentes em ambas modalidades, $12,2 \%$ nas internações domiciliares e $15,0 \%$ nos procedimentos domiciliares ambulatoriais. Lesões por envenenamento e outras causas externas corresponderam a $11,6 \%$ das internações domiciliares e a $9,7 \%$ dos procedimentos domiciliares ambulatoriais; dentre as causas externas podem justificar o uso de $\mathrm{AD}$ as sequelas de acidentes que impossibilitam o deslocamento do paciente aos serviços de saúde. Aliás, incapacidade comum às patologias neurológicas e crônicas, que exigem um cuidado longitudinal e contínuo. Por sua vez, os transtornos mentais e comportamentais foram motivo de $3,1 \%$ dos procedimentos domiciliares ambulatoriais, ausente dentre as internações domiciliares. E, ainda, as doenças osteomusculares e do tecido conjuntivo, representaram $2,4 \%$ dos procedimentos domiciliares ambulatoriais $(\mathrm{Ta}-$ bela 1). Estas não apareceram como motivo das internações domiciliares, mas estiveram presentes como diagnóstico secundário nesta modalidade, que podem estar relacionadas aos cuidados a ferimentos e úlceras de pressão, comum a pacientes com restrição de mobilidade.

Uma peculiaridade percebida na base de dados do SIA-SUS foi a inconsistência da informação de diagnóstico em 10,3\% (413.687) dos registros. Uma análise mais detalhada desta informação, possibilitou compreender que, nesta base de dados, o diagnóstico é registrado em três diferentes variáveis: CID principal, CID associado e CID-AD, esta última relacionada a situação ou agravo que motivou cada um dos atos/procedimentos destinados ao paciente, estando este registro completo. Portanto, disponibiliza somente o volume de produção, e exigindo expertise, conhecimento e manuseio maior dos dados disponíveis para se obter um perfil mais detalhado dos casos e cuidado prestado.

Com relação ao resultado do cuidado, a permanência ou prolongamento do cuidado nessa modalidade correspondeu à maioria dos resgistros em ambas modalidades, $53,1 \%$ nas internações domiciliares e $89,2 \%$ nos procedimentos domiciliares ambulatoriais (Tabela 1). A alta foi relevante na modalidade internação hospitalar $(31,8 \%)$, mas pouco relevante nos atendimentos domiciliares ambulatoriais, correspondendo a apenas $5,3 \%$, possivelmente dada a cronicidade da condição ou outra característica dos casos. Outros resultados, de forma discreta, foram registrados como transferência a outro tipo de serviço, admissão em serviço de urgência, internação domiciliar e enceramento administrativo. Os óbitos também foram pouco relevantes, ocorrendo em 1,6\% (1.571) das internações domiciliares e $1,7 \%$ (68.280) dos atendimentos domiciliares ambulatoriais (Tabela 1).

Outras informações possibilitam caracterizar o perfil de uso desses serviços. Referente aos atendimentos domiciliares ambulatoriais, o en- 
caminhamento à $\mathrm{AD} 1$, modalidade de cuidado de menor complexidade executada pelas equipes de Atenção Básica, ocorreu em 2,3\% dos casos. Foi possível, também, observar que a maior parte dos atendimentos $(93,3 \%)$ foi executado pelas EMAD, e apenas 6,7\% executados pelas EMAP. $\mathrm{E}$, ainda, que $63,3 \%$ das residências não eram cobertas por Estratégia Saúde da Família (ESF). Quanto a origem do paciente, $54,5 \%$ dos pacientes atendidos foram originados da Atenção Básica (AB), 23,8\% egressos de internação hospitalar, e apenas $5,7 \%$ de serviços de urgência.

No SIH-SUS, foram encontrados dentre outros, informações que complementaram a compreensão do funcionamento da $\mathrm{AD}$, como o tempo de permanência na internação domiciliar, onde $68,6 \%$ permaneceram neste tipo de cuidado por 29 dias ou mais, caracterizando a longitudinalidade deste tipo de cuidado. Já a média de permanência de cada paciente sob cuidado domiciliar ambulatorial foi de 26,6 dias, enquanto a média do número de procedimentos dispensados a cada paciente sob cuidado domiciliar ambulatorial foi de 8,5 , e a média de atendimentos/visitas recebidas por paciente foi de 1 (uma) visita durante o período aproximado de 1 (um) mês (26,6 dias).

Do volume total de procedimentos domiciliares ambulatoriais (4.008.612), os procedimentos mais frequentemente realizados foram as consultas, atendimentos e visitas. Em primeiro lugar: consulta/atendimento domiciliar (18,4\%), seguido pelos procedimentos de assistência domiciliar por equipe multiprofissional $(13,6 \%)$, assistência domiciliar por profissional de nível médio $(12,6 \%)$, visita domiciliar por profissional de nível médio (10,8\%), curativos $(10,4 \%)$ e atendimento fisioterapêutico (6,3\%). Nota-se, portanto, a predominância dos atendimentos prestados por equipes de profissionais de nível médio, em detrimento de equipes compostas por profissionais de nível superior e da atenção especializada, o que pode ter relação com o déficit na oferta de recursos humanos.

Em todo Brasil, 69 hospitais foram responsáveis por prover as 95.754 de internações domiciliares no período analisado. Em relação à natureza jurídica, 56 (81,2\%) eram hospitais públicos e $12(17,4 \%)$ eram privados não lucrativos. Apenas um hospital correspondia à privado lucrativo.

Em relação a distribuição geográfica, a região Nordeste concentrou o maior volume das internações domiciliares $(45,2 \%)$, seguida da região Sudeste $(22,5 \%)$ e região Centro-Oeste $(18,3 \%)$ (Tabela 1). Por último, com expressão reduzi- da, foi a região Norte (1,3\%). Embora a região Nordeste tenha abrigado o maior volume das internações domiciliares, esta modalidade esteve circunscrita, principalmente, ao estado do Ceará (Gráfico 1). Situação comum às outras regiões, cujo volume de internações domiciliares esteve circunscrito a determinadas Unidades da Federação (UF). A região Norte foi representada apenas pelo Pará, a Sudeste teve a maioria das internações domiciliares registradas em São Paulo, a região Sul no Rio Grande do Sul, e a região Centro-Oeste apenas foi representada pelo Distrito Federal (Gráfico 1).

A assistência domiciliar ambulatorial mostrou-se mais difundida pelas regiões geográficas, contemplando um maior número de UF por região. Todavia, há também diferenças marcantes em relação ao número de procedimentos utilizados entre as UF, e nessas, dentre seus municípios e regiões de saúde. Mais da metade dos procedimentos foram realizados na região Sudeste $(53,3 \%)$, seguido da região Nordeste $(26,0 \%)$ e região Sul $(11,4 \%)$ (Tabela 1). As regiões Centro -Oeste e Norte tiveram pouca representatividade com 5,2\% e 3,9\%, respectivamente (Tabela 1).

\section{AD no âmbito das Regiões de Saúde}

A constituição de região de saúde, pelo Decreto $\mathrm{n}^{\circ} 7.508$, busca ofertar cuidado de proximidade nas distintas modalidades assistenciais. Nesse contexto, o mapeamento da oferta dessa modalidade de cuidado é relevante, sobretudo pela discussão corrente sobre o processo de reorganização do SUS em regiões de saúde intra UF ${ }^{19}$.

Assim, a distribuição geográfica da produção agregada, considerando todos os anos de estudo, da AD no SUS no Brasil, por regiões de saúde ilustra que ambas modalidades de cuidado domiciliar em saúde (internação domiciliar e assistência domiciliar ambulatorial) estiveram circunscritas a determinadas áreas. Isso reforça a ideia de que o acesso a esse tipo de cuidado é geograficamente desigual, em todos os níveis geográficos: grandes regiões geográficas, UF, municípios e regiões de saúde (Figuras 1 e 2). Na internação domiciliar (Figura 1), por sua complexidade e desafios regionais específicos, como aqueles da região Norte, há vazios de oferta relativamente maiores que na assistência domiciliar ambulatorial (Figura 2). Contudo, esses vazios assistenciais não são inerentes a $\mathrm{AD}$, os desafios para superar insuficiência da oferta e regionalização de serviços estão presentes em outros tipo de cuidado, além de outros setores. 


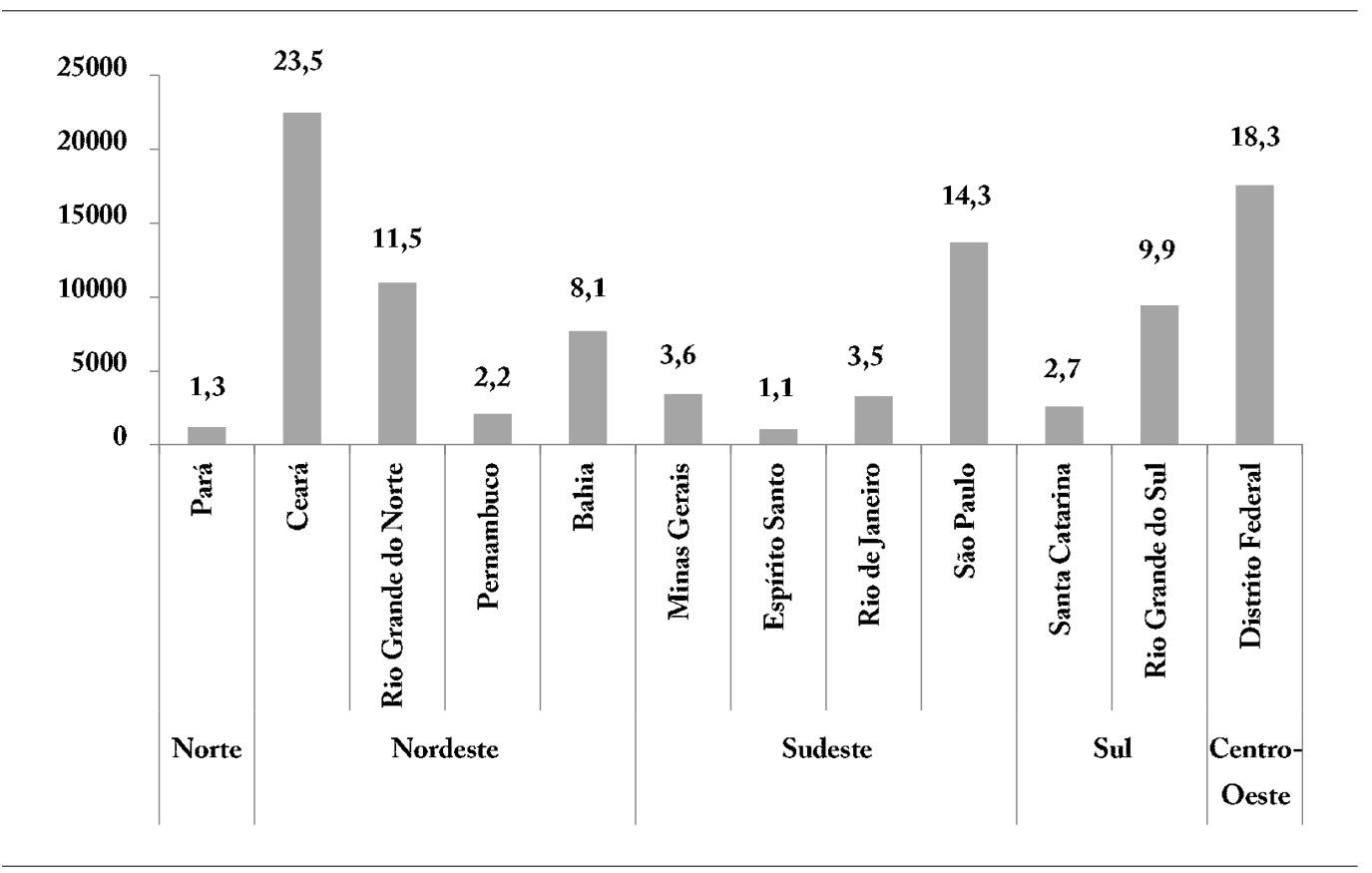

Gráfico 1. Distribuição percentual de internações domiciliares por Unidade da Federação (UF) e grande região geográfica. Brasil, 2008-2016.

Fonte: Ministério da Saúde: Sistema de Informações Hospitalares (SIH/SUS) - Movimento de AIH.

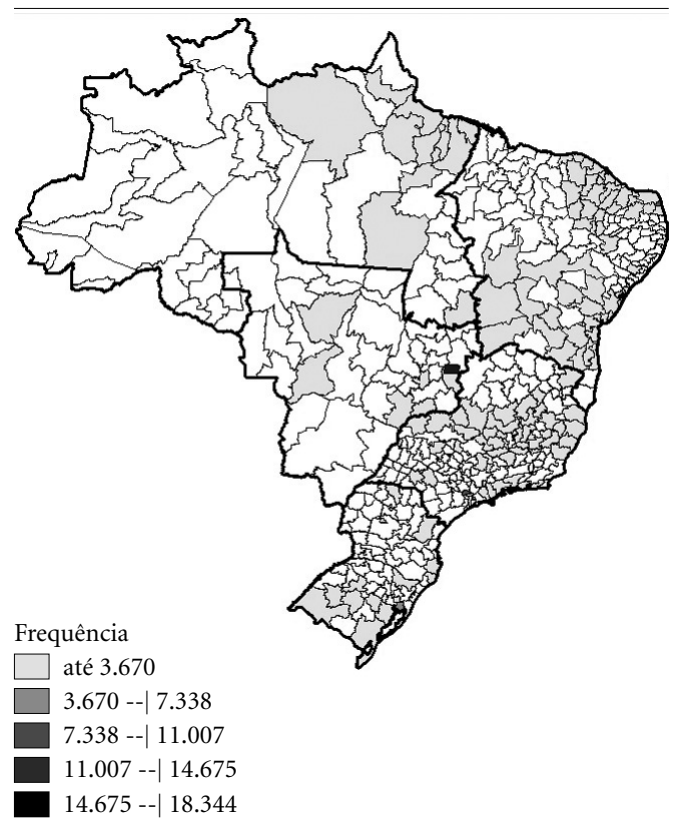

Figura 1. Mapa da distribuição das internações domiciliares por regiões de saúde. Brasil, 2008-2016.

Fonte: Ministério da Saúde: Sistema de Informações Hospitalares (SIH/SUS) - Movimento de AIH.

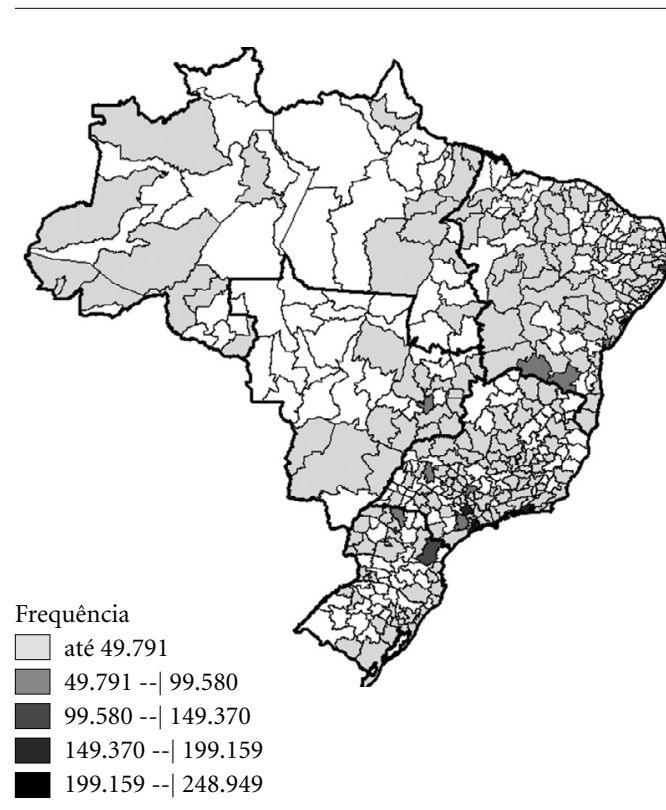

Figura 2. Mapa da distribuição dos procedimentos domiciliares ambulatoriais por regiões de saúde. Brasil, 2012-2016.

Fonte: Ministério da Saúde: Sistema de Informações Ambulatoriais (SIA/SUS) - Serviço de Atenção Domiciliar. 
Observando a distribuição geográfica de ambas modalidades de $\mathrm{AD}$ - internação domiciliar, prestada por hospitais executores deste serviço, e assistência domiciliar ambulatorial, prestada pelos $\mathrm{SAD}$, nas suas respectivas equipes na modalidade $\mathrm{AD} 1$ (na $\mathrm{AB}$ ) e nas modalidades AD2 e AD3 (pelas EMAD e EMAP) -, ficou evidente que foram concentrados nas regiões Sudeste e Nordeste (Figuras 1 e 2). Estando o maior volume de atendimentos circunscritos em determinadas Regiões de Saúde. As Regiões de Saúde do Sudeste contemplam mais da metade dos procedimentos ambulatoriais realizados pelos SAD (Figura 2), enquanto que as Regiões de Saúde do Nordeste abrigaram quase a metade das internações domiciliares (Figura 1), com detaque para o Ceará.

Assim, o mapeamento da $\mathrm{AD}$ possibilitou perceber, de maneira abrangente, a existência de grandes diferenças regionais, tanto em relação ao volume de serviços e procedimentos, quanto em relação à variação das modalidades. A maior parte dos serviços e procedimentos no âmbito da AD encontram-se circunscritos a determinadas localidades. Isso parece indicar que, apesar de já haver um programa nacional que orienta a prestação dessa modalidade de cuidado, a implementação da $\mathrm{AD}$ continua restrita a algumas Secretarias de Saúde Municipais e Estaduais. Assim, esse programa, até o período analisado, não ganhou amplitude nacional, caracterizando uma marcante desigualdade regional.

\section{Conclusão}

Reconhecendo o caráter exploratório e descritivo do estudo, a principal limitação é decorrente da fonte de informação utilizada, sobretudo quanto a subnotificação de algumas informações e diferentes unidades de observação das bases de dados: internação para o SIH-SUS e procedimentos realizados para SIA-SUS. Apesar das limitações, a disponibilidade de informações nos SIS-SUS superou a expectativa inicial, possíbilitando traçar o perfil da atenção domiciliar no SUS. Em linhas gerais, o SIH-SUS vem sendo mais utilizado, em comparação ao SIA-SUS, em estudos desde a década de 1990, sendo que com base no SIA há pouca experiência acumulada.

$\mathrm{Na} A D$ percebe-se a importância do cuidador familiar para a qualidade do cuidado domiciliar, bem como o ônus do desempenho desta função mencionados em estudos relacionados a sobrecarga e estratégias de apoio aos cuidadores familiares $^{3,20,21}$. Vale salientar que as bases de dados não contemplam informação a esse respeito. No entanto, informações sobre o nível de autonomia do paciente, do seu ambiente familiar, estrutura física do domicílio e a respeito do cuidador, contribuiriam para conhecimento das singularidades e necessidades de tal aporte no cuidado em saúde.

Por sua vez, a capacitação profissional voltada à compreensão desta nova forma de cuidar, assim como suas especificidades técnicas, despontam como uma necessidade atrelada à consolidação da $\mathrm{AD}$ enquanto um modelo mais fundamentado de atenção à saúde. No campo da organização e coordenação da atenção domiciliar no contexto brasileiro, não é notória, na prática,que haja a articulação formal e contínua entre as EMAD e EMAP com demais serviços de saúde. As equipes de Saúde da Família (eSF) e Núcleo de Apoio à Saúde da Família (NASF) desempenham, sim, as atividades da $\mathrm{AD} 1$, porém de maneira incorporada aos seus processos de trabalho, não destacando a existência dessa conformação exposta na legislação e na produção analisada nos SIS-SUS. Tal como evidenciado nos resultados, observouse que na maioria das localidades nas quais foi registrada a presença das EMAD e EMAP não havia cobertura por ESF.

Preliminarmente foi, também, possível vislumbrar a internação domiciliar como um cuidado continuado da assistência hospitalar, enquanto a assistência domiciliar ambulatorial parece mais próxima de um cuidado substitutivo ou complementar à assistência ambulatorial. Esta última apresenta-se de uma maneira mais delineada, constituindo um programa específico - o Programa Melhor em Casa. Todavia, a internação domiciliar não é contemplada nos manuais instrutivos deste Programa, mostrando-se a parte dele.

Pouco trabalhando na literatura nacional, conhecer a $\mathrm{AD}$ baseada na legislação e dados secundários sobre a produção dessa modalidade de cuidado no nível nacional foi objetivo desse estudo. Os achados permitiram um diagnóstico inicial dos avanços e dos nós críticos à sua expansão. Contudo, limites no sistema de informação existem e até mesmo merecem recursos que permitam aprofundar o panorama aqui delineado. $\mathrm{O}$ acompanhamento e monitoramento da sua evolução e entraves no tocante à expansão, tanto em abrangência territorial, quanto em relação à inserção e consolidação como modalidade de atenção na RAS se faz necessário. Para tal, é ainda essencial se conhecer o volume de cuidado domiciliar que é prestado no âmbito do Programa de Saúde da Família, até o momento não registrado no SIA. 
Adicionalmente, muitos desafios concorrem para o desempenho do cuidado no domicílio, como a construção de plano de cuidados, o preparo dos profissionais em saúde para assumir a atribuição de cuidar de forma integral, inserido no contexto de vida do usuário, o trabalho em equipe. Em se tratando do SUS, levando-se em consideração a desigualdade regional e abrangência territorial, esse quadro requer mais atenção, exigindo a retaguarda da rede de serviços com prontidão para garantir acesso e continuidade do cuidado no tempo oportuno.

\section{Colaboradores}

FL Rajão participou de todas as etapas de desenvolvimento do estudo e elaboração deste artigo. M Martins participou da concepção, análise dos dados, redação e revisão crítica do documento.

\section{Agradecimentos}

À Coordenação de Aperfeiçoamento de Pessoal de Nível Superior - CAPES (Bolsa de Mestrado), ao Conselho Nacional de Desenvolvimento Científico e Tecnológico (CNPq), ao Programa de Pós-graduação em Saúde Pública da Escola Nacional de Saúde Pública Sérgio Arouca da Fundação Oswaldo Cruz.
Em suma, este trabalho almejou revelar como vem sendo consolidada a AD no SUS, sobretudo, buscando contribuir para o reconhecimento dos entraves que possam minimizar as desigualdades geográficas na oferta dessa modalidade. O perfil demográfico e epidemiológico coloca como missão o cuidar de múltiplas doenças crônicas em períodos contínuos. Assim, o quadro descrito mapeou uma AD circunscrita a algumas localidades, descrevendo os recursos disponíveis para atender essas demandas de cuidado, crescentes no contexto brasileiro e mundial.

\section{Referências}

1. Braga PP, Sena RR, Seixas CT, Castro EAB, Andrade AM, Silva YC. Oferta e demanda na atenção domiciliar em saúde. Cien Saude Colet 2016; 21 (3):903-912.

2. Feuerwerker LCM, Merhy EE. A contribuição da atenção domiciliar para a configuração das redes substitutivas de saúde: desinstitucionalização e transformação de práticas. Rev Pan-Am Salud Publica 2008; 24(3):180-188.

3. Creutzberg M, Santos BRL. Famílias cuidadoras de pessoas idosas: relação com instituições sociais e de saúde. Rev Bras Enferma 2003; 56(6):624-629.

4. Kerber NPC, Kirchhof ALC, Cezar-Vaz MR. Considerações sobre a atenção domiciliária e suas aproximações com o mundo do trabalho na saúde. Cad Saude Publica 2008; (24)3:485-493.

5. Loyola CMD. Cuidado Continuado. In: Giovanella L, Escore 1S, Lobato LVC, Noronha JC, Carvalho AI, organizadores. Políticas e Sistema de Saúde no Brasil. Rio de Janeiro: Editora Fiocruz; Centro Brasileiro de Estudos de Saúde; 2008. p. 959-978.

6. Brasil. Ministério da Saúde (MS). Secretaria de Atenção à Saúde. Departamento de Atenção Básica. Caderno de Atenção Domiciliar. Volume 1. Brasília: MS; 2012.

7. Faria L, Calábria LKL, Amaral S. Sentimento mútuo: as relações de cuidar e ser cuidado no domicílio. In Faria L, Calábria LKL, Alves WA, organizadores. Envelhecimento, um olhar interdisciplinar. São Paulo: Editora Hucitec; 2016. p. 61-88. 
8. Gleen EN. Forced to Care. Coercion and Caregiving in America [livro na Internet]. Cambridge, Massachusetts, Londres: Harvard University Press; 2010 [acessado 2017 Mar 8]. Disponível em: https://caringlabor. files.wordpress.com/2012/05/forced-to-care.pdf

9. Lorincz CY, Drazen E, Sokol PE, Neerokonda KV, Metzquer J, Toepp MV, Maul L, Classen DC, Wynia MK. Research in ambulatory patient safety 2000-2010: a 10-year review [documento na Internet]. Chicago: AMA; 2011 [acessado 2019 Maio 17]. Disponível em: https://cdn.ymaws.com/npsf.site-ym.com/resource/ resmgr/PDF/Research-in-Amb-Pat-Saf_AMAr.pdf

10. Barber N. Should we consider non-compliance a medical error? Qual Saf Health Care 2002; 1(11):8184.

11. Stajduhar KI. Examining the perspectives of family members involved in the delivery of palliative care at home. J Palliat Care 2002; 1(19):27-35.

12. Lacerda MR, Giacomozzi CM, Oliniski SR, Truppel TC. Atenção à Saúde no Domicílio: modalidades que fundamentam sua prática. Saude Soc 2006; 2(15):8895.

13. Rajão FL. Atenção Domiciliar no Brasil: estudo exploratório sobre marco legal, consolidação e utilização de serviços no âmbito do SUS [dissertação]. Rio de Janeiro: Escola Nacional de Saúde Pública, Fiocruz; 2018.

14. Brasil. Ministério da Saúde (MS). Secretaria de Atenção à Saúde. Departamento de Atenção Básica. Caderno de Atenção Domiciliar. Volume 2. Brasília: MS; 2013.

15. Brasil. Ministério da Saúde (MS). Secretaria de Atenção à Saúde. Departamento de Atenção Básica. Manual de Monitoramento e Avaliação do Programa Melhor em Casa. Brasília: MS; 2014.

16. Brasil. Ministério da Saúde (MS). Secretaria de Atenção à Saúde. Departamento de Atenção Básica. Caderno de Atenção Domiciliar. Volume 3. Brasília: MS; 2015.

17. Faller JW, Brusnicki PH, Zilly A, Brofman, MCBFS, Cavalhieri L. Perfil de idosos acometidos por câncer em cuidados paliativos em domicílio. Rev Kairós Gerontol 2016; 2(19):22-43.

18. Muniz EA, Aguiar MFS, Brito MCC, Freitas CASL, Moreira ACA, Araújo CRC. Desempenho das atividades de vida diária de idosos em Atenção Domiciliar na Estratégia Saúde da Família. Rev Kairós Gerontol 2016; 2(19):133-146.

19. Brasil. Ministério da Saúde (MS). Decreto no 7.508, de 28 de junho de 2011. Regulamentação da Lei no 8.080/90. Diário Oficial da União; 2013.

20. Carvalho LC. A disputa de planos de cuidado na atenção domiciliar [dissertação]. Rio de Janeiro: Univesidade Federal do Rio de Janeiro; 2009.

21. Karsch UM. Idosos dependentes: famílias e cuidadores. Cad Saude Publica 2003; 3(19):861-866.

22. Brasil. Ministério da Saúde (MS). Agência Nacional de Vigilância Sanitária (Anvisa). Resolução RDC no 11, de 26 de janeiro de 2006. Diário Oficial da União; 2006.
23. World Health Organization (WHO). Home-based and long-term care: home care issues at the approach of the 21th century from a World Health Organization perspective - a literature review. Genebra: WHO; 1999.

24. Brasil. Ministério da Saúde (MS). Secretaria de Atenção à Saúde. Departamento de Atenção Hospitalar e de Urgência. Segurança do paciente no domicílio. Brasília: MS; 2016.

25. Wealth Canada. Policy and Consultation Branch. National health expenditures in Canada 1975-1996: fact sheets [página na Internet]. Ottawa: Wealth Canada; 1997 [acessado 2006 Jul 7]. Disponível em: http://socialpolicy.ca/cush/m7/healthfact.pdf

26. Ryu H, An J, Koabyashi M. Home health nursing care agenda based on health policy in Korea. Nurs Health Sci 2005; 7(2):92-98.

27. Thomé B, Dykes AK, Hallberg IR. Home care with regard to definition, care recipients, content and outcome: systematic literature review. J Clin Nurs 2003; 12(6):860-872.

28. Ribeiro CA. Assistência domiciliar: uma 'nova' modalidade de atenção à saúde [página na Internet]. [acessado 2004 Ago 5]. Disponível em: http://www.unimeds. com.br/conteudo/ME01.htm

29. Mazza MMPR. A visita domiciliária como instrumento de assistência de saúde [página na Internet]. [acessado 2004 Jun 1]. Disponível em: http://www.fsp.usp.br/ MAZZA.htm

30. Freitas AV, Bittencourt CM, Tavares JL. Atuação da enfermagem no serviço de internação domiciliar: relato de experiência. Rev Baiana Enferm 2000; 13(1/2):103107.

31. Murashima S, Nagata S, Magilvy JK, Fukui S, Kayama M. Home care nursing in Japan: a challenge for providing good care at home. Public Health Nurs 2002; 19(2):94-103.

32. Lacerda MR. Tornando-se profissional no contexto domiciliar: vivência do cuidado da enfermeira [tese]. Florianópolis: Universidade Federal de Santa Catarina; 2000.

33. 33. Nogueira JM. Enfermos crônicos domiciliares: valorização integral físico-cognitiva e caídas durante 3 anos de seguimento. Atencion Primária 2000; 25(3):16-17.

Artigo apresentado em 30/05/2019

Aprovado em 07/08/2019

Versão final apresentada em 28/11/2019 